\title{
A INFLUÊNCIA DA IDADE NA AQUISIÇÃO DE SEIS VOGAIS DO INGLÊS POR ALUNOS BRASILEIROS
}

THE INFLUENCE OF AGE ON THE ACQUISITION OF SIX ENGLISH VOWELS BY BRAZILIAN LEARNERS

Ronaldo Mangueira Lima Júnior ${ }^{1}$

Resumo: Foram analisadas acusticamente as produções das vogais [i: I $\varepsilon æ$ $\mathrm{u}: \mathrm{v}$ de brasileiros que estavam cursando o último semestre de seus cursos de inglês, que começaram a estudar a língua em diferentes idades e que nunca moraram ou estudaram inglês fora do Brasil. Os seus espaços vocálicos, com base nos valores de F1 e F2, foram comparados ao espaço vocálico de 10 falantes nativos de inglês. Os resultados mostraram que os alunos brasileiros tendem a agrupar os pares de vogais [i: $\mathrm{I}$ ], [ $\varepsilon$ æ] $e$ [u: $v$ ] em uma única categoria fonológica prototípica influenciada pelo português, i.e. [i], $[\varepsilon] e[\mathrm{u}]$, respectivamente. Essa tendência foi encontrada mesmo entre aprendizes mais novos e tendeu a se acentuar com o aumento da idade de início de aquisição, mostrando que a idade é um fator que influencia, porém não determina, a aquisição das vogais do inglês por alunos brasileiros.

Palavras-chave: aquisição fonológica; inglês-L2; vogais.

Abstract: The production of the English vowels [i: I $\varepsilon$ u: $v$ ] by Brazilian learners who were in the last semester of their English courses, had begun studying English at different ages and had never lived nor studied English abroad was acoustically analyzed. Their vowel spaces, based on F1 and F2 values, were compared to those of 10 native speakers of English. The results showed that the Brazilian learners tend to group the pairs of vowels [i: $\mathrm{I}$, [ $\varepsilon$ $æ]$ and $[\mathrm{u}: \mathrm{v}$ ] into one single prototypical phonological category influenced by Portuguese, i.e. [i], $[\varepsilon]$ and $[\mathrm{u}]$, respectively. This tendency was clear even among the younger learners and it tended to augment with the increase of age of onset, showing that the age factor influences, yet does not determine, the acquisition of English vowels by Brazilian learners.

Keywords: phonological acquisition; English as a foreign language; vowels.

1 Doutor em Linguística pela UNB. Professor adjunto da Universidade Federal do Ceará. 


\section{Introdução}

Uma das expectativas de aprendizes de línguas, principalmente daqueles que procuram por cursos particulares, é de aprender a falar a língua estrangeira (L2). O uso do verbo falar na pergunta "você fala inglês?", por exemplo, revela a ênfase que há na expectativa do domínio da oralidade quando se estuda uma L2. A comunicação oral efetiva, por sua vez, necessita de uma inteligibilidade mínima na pronúncia. São inúmeros os exemplos em forma de anedota de situações frustrantes, e até constrangedoras, pelas quais aprendizes de uma língua podem passar por não terem o domínio necessário de sua fonologia. Kelly (2006), por exemplo, imagina um aprendiz em um restaurante pedindo soap [ $\mathrm{so}^{\circ} \mathrm{p}$ ] em vez de soup [su:p], devido à falta de controle da pronúncia. Celce-Murcia, Brinton e Goodwin (1996) imaginam um turista nos Estados Unidos pedindo batter [bætər] em vez de butter [bıtər] e recebendo, no lugar de manteiga, um olhar estranho. Fortes problemas de pronúncia podem até mesmo distanciar falantes nativos do aprendiz de L2 (PARRINO, 1998) ou levá-los a evitar futuras interações com os usuários da L2 (SINGLETON; RYAN, 2004).

É por isso que o mercado de ensino de língua estrangeira, principalmente o inglês, oferece cursos para crianças a partir da mais tenra idade, uma vez que a dificuldade de adquirir os padrões fonológicos de uma L2 tende a aumentar com o avanço da idade (e.g. BIALYSTOK; HAKUTA, 1999; BONGAERTS, 2005; ELLIS, 2008; FLEGE, 1999; HAKUTA, 2001; LEATHER, 2003; MOYER, 2004; SINGLETON; RYAN, 2004). O problema não é que as pessoas perdem a capacidade de aprender novas pronúncias, e sim o fato de terem aprendido o sistema fonológico de suas L1s tão bem (FLEGE, 1999; LEATHER, 2003). Na aquisição da L1, as crianças precisam aprender a agrupar exemplares de uma mesma palavra - foneticamente diferentes devido a variações idiossincráticas e regionais dos falantes - dentro de uma mesma categoria fonológica. Para tal, os cérebros de bebês adquirindo uma L1 tomam notas estocásticas do que ouvem para formarem protótipos fonológicos capazes de arcar com a variação fonética do que ouvem (BYBEE, 2003; CRISTÓFARO-SILVA, 2003; KUHL, 1991; 1993; KUHL et al., 2008).

Consequentemente, com o amadurecimento e a fixação do sistema fonológico da L1, torna-se mais difícil identificar os sons de uma L2 que são muito próximos a sons da L1. Os sons que se contrastam menos são os mais difíceis de aprender e dominar, uma vez que os aprendizes tendem a não percebê-los como diferentes. Sendo assim, um aprendiz de L2 adulto, 
por não perceber o som da L2 como diferente de um próximo de sua L1, acaba classificando-o em uma das categorias fonológicas (prototípicas) de sua L1 (FLEGE, 1995; 1999; 2007).

Entretanto, há uma lacuna nas pesquisas sobre a influência da idade na aquisição fonológica de L2 quando se aprende a L2 exclusivamente em salas de aula, sem imersão, e, consequentemente, com exposição à L2 muito menor do que quando se adquire a L2 em imersão. Este estudo visou a colaborar com o preenchimento dessa lacuna ao investigar a aquisição das vogais [i: i $\varepsilon$ æ u: $\mho$ ] por aprendizes brasileiros que começaram seus cursos de inglês em diferentes idades, exclusivamente em salas de aula do Brasil.

A pergunta de pesquisa foi "qual é a influência, se alguma, que o fator 'idade no início da aquisição' tem na aquisição das vogais [i: I $\varepsilon$ æ u: v] entre alunos brasileiros que começaram o curso de inglês em idades diferentes?". Essas vogais foram escolhidas por sua conhecida dificuldade para alunos brasileiros, que tendem a assimilar os pares [i: $\mathrm{I}],[\varepsilon æ] \mathrm{e}[\mathrm{u}: \mathrm{v}]$ para as categorias prototípicas $[\mathrm{i}],[\varepsilon]$ e $[\mathrm{u}]$ do português, respectivamente. A principal hipótese é a de que conforme as idades de início do curso de inglês aumentam, maiores são os efeitos da transferência dos aspectos fonético-fonológicos da L1 para a L2, causando menor distinção entre os pares de vogais [i: $\mathrm{i}],[\varepsilon æ]$ e $[\mathrm{u}: v]$.

\section{Metodologia}

A pesquisa, aprovada pelo Comitê de Ética em Pesquisa do Instituto de Ciências Humanas da Universidade de Brasília sob o número 02-11/2011, foi conduzida com alunos de um instituto particular de ensino de língua inglesa, no qual os alunos estudam a L2 como atividade extracurricular. A escola tem cursos para alunos a partir dos quatro anos de idade. As aulas são conduzidas em inglês, independentemente do nível e da idade dos alunos; o uso da L1 por parte dos alunos é desencorajado e o uso da L2 é enfatizado; os professores têm alto nível de proficiência em inglês, precisando, além de apresentar certificação internacional de proficiência em inglês, passar por uma extensa prova escrita e entrevista oral para que a contratação seja cogitada. Por contar com mais de 100 professores, a escola consegue trocar o professor das turmas semestralmente, possibilitando a exposição dos alunos a diferentes sotaques e dialetos.

Participaram da coleta de dados 30 alunos, todos cursando o último semestre do curso de inglês avançado. Os alunos que participaram da Organon, Porto Alegre, v. 30, n. 58, p. 15-31, jan/jun. 2015. 
pesquisa estudaram exclusiva e ininterruptamente nessa instituição, e obtiveram notas acima da média de aprovação durante seus estudos. Além disso, nenhum dos participantes morou fora do Brasil ou passou mais que quatro semanas consecutivas a turismo em um país falante de inglês, pois o objetivo era o de investigar a aquisição fonológica de alunos que adquiriram a L2 exclusivamente no Brasil por meio de instrução explícita.

Os participantes foram divididos em três grupos: 10 participantes que iniciaram os seus estudos de inglês-L2 no Brasil antes dos 12 anos de idade, 10 que iniciaram o estudo entre os 12 e os 14 anos de idade, e 10 que iniciaram seus estudos após os 16 anos de idade, sendo cada grupo composto por cinco homens e cinco mulheres. Os grupos serão denominados G1, G2 e G3 para facilitar a referência, e suas informações estão na tabela a seguir.

\begin{tabular}{|l|c|c|c|c|}
\hline Grupo & & G1 & G2 & G3 \\
\hline Quantidade de participantes & & 10 & 10 & 10 \\
\hline $\begin{array}{l}\text { Idade de início dos estudos } \\
\text { de inglês-L2 no Brasil }\end{array}$ & & $\begin{array}{c}\text { Antes de 12 } \\
\text { (entre 8 e 11) }\end{array}$ & Entre 12 e 14 & $\begin{array}{c}\text { Após 16 } \\
\text { (entre 17 e 55) }\end{array}$ \\
\hline \multirow{2}{*}{ Idade no início do curso } & $\mathrm{M}$ & 9,3 & 12,6 & 36 \\
\cline { 2 - 5 } & $\mathrm{DP}$ & 0,9 & 0,6 & 13 \\
\hline \multirow{2}{*}{ Idade na gravação } & $\mathrm{M}$ & 16,4 & 18,6 & 44,5 \\
\cline { 2 - 5 } & $\mathrm{DP}$ & 1 & 1,3 & 13 \\
\hline $\begin{array}{l}\text { Tempo (em anos) cursando } \\
\text { inglês }\end{array}$ & $\mathrm{M}$ & 7,1 & 5,8 & 5,3 \\
\cline { 2 - 5 } & $\mathrm{DP}$ & 0,7 & 0,9 & 1,4 \\
\hline
\end{tabular}

Fig. 1: Participantes da pesquisa

Além dos aprendizes, 10 falantes nativos de inglês americano, cinco homens e cinco mulheres, também foram gravados e compuseram o grupo controle. Os falantes nativos tinham entre 18 e 74 anos de idade (média 38,7; DP 20). Foram escolhidos falantes nativos americanos, pois essa é a variedade de inglês ensinada na instituição de origem dos alunos participantes da pesquisa. Apesar de falantes nativos de regiões diferentes gerarem dados acústicos com maior variação, seus dados podem servir como base comparativa mais eficiente, visto que alunos brasileiros de inglês-L2 não têm como alvo uma variedade regional específica do inglês americano.

Cada participante da pesquisa foi gravado lendo palavras-alvo inseridas em uma frase veículo, objetivando a coleta de dados para a análise acústica da produção das vogais anteriores [i: I $\varepsilon$ æu: $\circlearrowright$ ] em posição tônica. O corpus foi constituído de três tokens para cada vogal. Cada token foi uma palavra com o contexto fonológico CVC, sendo ambos os Cs consoantes oclusivas surdas. Um único contexto fonológico possibilitou isolar as va- 
riações acústicas advindas de diferentes contextos fonológicos. A escolha de oclusivas surdas foi feita para facilitar a segmentação da vogal, que foi realizada no programa PRAAT, versão 5.2.25 (BOERSMA; WEENINK, 2011). Para as vogais anteriores, foi possível encontrar quartetos mínimos, o que controla mais ainda o contexto no qual as vogais estão inseridas. Para as vogais posteriores, entretanto, não há tantos pares mínimos em inglês, então foram escolhidos tokens que deixassem a vogal em um contexto consonantal próximo ao das outras.

\begin{tabular}{|c|c|c|c|c|c|}
\hline$[\mathbf{i}:]$ & {$[\mathbf{I}]$} & {$[\boldsymbol{\varepsilon}]$} & {$[\mathfrak{x}]$} & {$[\mathbf{u}:]$} & {$[\boldsymbol{\sigma}]$} \\
\hline peak & pick & peck & pack & boot & book \\
\hline Pete & Pitt & pet & pat & poop & put \\
\hline teak & tick & tech & tack & toot & took \\
\hline
\end{tabular}

Fig. 2: Corpus para coleta de dados para análise acústica

Os tokens foram apresentados aos participantes na frase-guia "I said token this time", também utilizada por Watkins e Rauber (2010). As frases foram apresentadas aos alunos em uma apresentação de Powerpoint, com uma frase em cada slide.

Cada token foi apresentado quatro vezes de maneira aleatória. Dessa maneira, com três tokens para cada uma das seis vogais, e cada token sendo analisado quatro vezes, houve 72 vogais para cada participante, 2.160 vogais de aprendizes, 720 vogais do grupo controle, 2.880 vogais totais.

Para as gravações, foram utilizados uma placa de som Roland Tri Capture e um microfone dinâmico, unidirecional, supercardióide AKG-P5S. $\mathrm{O}$ áudio foi capturado com o programa Audacity 1.3.10 em mono, com taxa de amostragem de $44.000 \mathrm{~Hz}$, e salvo em arquivos wav. Apesar de não haver tratamento acústico, os locais das gravações foram escolhidos considerando-se a necessidade em obter o mínimo de ruído possível para uma gravação com fins de análise acústica.

As vogais foram segmentadas no programa PRAAT versão 5.2.25 (BOERSMA; WEENINK, 2011). Os pontos de início e fim das vogais considerados foram o primeiro e último vale do pulso periódico na onda que contivesse amplitude considerável, que se assemelhasse ao período vocálico, e que apresentassem formantes estáveis no espectrograma.

Um dos métodos mais comumente utilizados para extração dos formantes é o LPC (Linear Predictive Coding), que é um algoritmo preditivo que decompõe o sinal acústico fazendo uma estimativa das ressonâncias geradas no trato vocálico. Todavia, a análise automática de LPC é criticada (e.g. VALLABHA; TULLER, 2002; WEMPE; BOERSMA, 2003) porque o pesquisador 
deve definir de antemão parâmetros de análise, como a ordem do LPC, i.e. a quantidade de formantes a ser encontrada, e a frequência máxima para os formantes. O problema é que estabelecer parâmetros para análises automáticas introduz erros sistemáticos na estimativa dos formantes, com o LPC estimando picos que não existem ou deixando de estimar picos que existem.

Uma solução é conferir o ajuste do LPC ao espectro de FFT (obtido pelo cálculo do algoritmo Fast Fourier Transform) vogal por vogal. Essa conferência, apesar de consumir mais tempo e ser mais trabalhosa, permite ajustar, quando necessário, a frequência máxima ou a ordem do LPC. É exatamente isso que permitem os scripts utilizados para a extração de F1, F2 e F3 nesta pesquisa (ARANTES, 2010; 2011).

Após extração, os valores de F1 e F2 dos participantes foram normalizados pelo método de Watt e Fabricius (2002). A normalização das vogais, assim como as plotagens de seus espaços vocálicos no plano F1 x F2, foram realizadas com o pacote vowels (KENDALL; THOMAS, 2009) para o programa R versão 2.15.1 (R CORE TEAM, 2012).

Para a extração dos valores de duração, foi utilizado o script de Arantes (2008).

\section{Resultados}

A tabela a seguir apresenta as médias e desvios-padrão de F1 e F2 (em Hertz) de cada vogal para cada grupo após a normalização.

\begin{tabular}{|c|c|c|c|c|c|c|c|}
\hline \multirow{2}{*}{ Grupo } & \multirow{2}{*}{ Formantes } & \multicolumn{6}{|c|}{ Vogais } \\
\hline & & [i:] & {$[\mathrm{I}]$} & {$[\varepsilon]$} & [æ] & [u:] & {$[\mho]$} \\
\hline \multirow{2}{*}{ Controle } & F1 & $\begin{array}{c}294 \\
(\mathrm{DP}=19)\end{array}$ & $\begin{array}{c}394 \\
(\mathrm{DP}=30)\end{array}$ & $\begin{array}{c}513 \\
(\mathrm{DP}=32)\end{array}$ & $\begin{array}{c}624 \\
(\mathrm{DP}=41)\end{array}$ & $\begin{array}{c}318 \\
(\mathrm{DP}=26)\end{array}$ & $\begin{array}{c}411 \\
(\mathrm{DP}=30)\end{array}$ \\
\hline & $\mathrm{F} 2$ & $\begin{array}{c}1941 \\
(\mathrm{DP}=36)\end{array}$ & $\begin{array}{c}1710 \\
(\mathrm{DP}=54)\end{array}$ & $\begin{array}{c}1599 \\
(\mathrm{DP}=76)\end{array}$ & $\begin{array}{c}1539 \\
(\mathrm{DP}=65)\end{array}$ & $\begin{array}{c}1366 \\
(\mathrm{DP}=197)\end{array}$ & $\begin{array}{c}1284 \\
(\mathrm{DP}=106)\end{array}$ \\
\hline \multirow{2}{*}{ G1 } & F1 & $\begin{array}{c}324 \\
(\mathrm{DP}=28)\end{array}$ & $\begin{array}{c}353 \\
(\mathrm{DP}=34)\end{array}$ & $\begin{array}{c}557 \\
(\mathrm{DP}=44)\end{array}$ & $\begin{array}{c}565 \\
(\mathrm{DP}=47)\end{array}$ & $\begin{array}{c}345 \\
(\mathrm{DP}=35)\end{array}$ & $\begin{array}{c}368 \\
(\mathrm{DP}=30)\end{array}$ \\
\hline & F2 & $\begin{array}{c}1931 \\
(\mathrm{DP}=52)\end{array}$ & $\begin{array}{c}1857 \\
(\mathrm{DP}=91)\end{array}$ & $\begin{array}{c}1632 \\
(\mathrm{DP}=79)\end{array}$ & $\begin{array}{c}1641 \\
(\mathrm{DP}=91)\end{array}$ & $\begin{array}{c}1183 \\
(\mathrm{DP}=184)\end{array}$ & $\begin{array}{c}1203 \\
(\mathrm{DP}=129)\end{array}$ \\
\hline \multirow{2}{*}{ G2 } & F1 & $\begin{array}{c}336 \\
(\mathrm{DP}=18)\end{array}$ & $\begin{array}{c}352 \\
(\mathrm{DP}=27)\end{array}$ & $\begin{array}{c}541 \\
(\mathrm{DP}=28)\end{array}$ & $\begin{array}{c}539 \\
(\mathrm{DP}=24)\end{array}$ & $\begin{array}{c}359 \\
(\mathrm{DP}=23)\end{array}$ & $\begin{array}{c}375 \\
(\mathrm{DP}=41)\end{array}$ \\
\hline & F2 & $\begin{array}{c}1916 \\
(\mathrm{DP}=59)\end{array}$ & $\begin{array}{c}1882 \\
(\mathrm{DP}=59)\end{array}$ & $\begin{array}{c}1644 \\
(\mathrm{DP}=70)\end{array}$ & $\begin{array}{c}1642 \\
(\mathrm{DP}=60)\end{array}$ & $\begin{array}{c}1201 \\
(\mathrm{DP}=171)\end{array}$ & $\begin{array}{c}1245 \\
(\mathrm{DP}=183)\end{array}$ \\
\hline \multirow{2}{*}{ G3 } & F1 & $\begin{array}{c}337 \\
(\mathrm{DP}=35)\end{array}$ & $\begin{array}{c}339 \\
(\mathrm{DP}=29)\end{array}$ & $\begin{array}{c}549 \\
(\mathrm{DP}=46)\end{array}$ & $\begin{array}{c}553 \\
(\mathrm{DP}=52)\end{array}$ & $\begin{array}{c}360 \\
(\mathrm{DP}=28)\end{array}$ & $\begin{array}{c}376 \\
(\mathrm{DP}=43)\end{array}$ \\
\hline & $\mathrm{F} 2$ & $\begin{array}{c}1926 \\
(\mathrm{DP}=58)\end{array}$ & $\begin{array}{c}1920 \\
(\mathrm{DP}=48)\end{array}$ & $\begin{array}{c}1708 \\
(\mathrm{DP}=67)\end{array}$ & $\begin{array}{c}1707 \\
(\mathrm{DP}=68)\end{array}$ & $\begin{array}{c}1174 \\
(\mathrm{DP}=144)\end{array}$ & $\begin{array}{c}1196 \\
(\mathrm{DP}=138)\end{array}$ \\
\hline
\end{tabular}

Fig. 3: Médias de F1 e F2 (em Hz) e desvio-padrão (DP) para cada grupo após normalização 
A figura 4 apresenta as plotagens das vogais de cada grupo com base nos dados da tabela anterior. O local de inserção de cada vogal na plotagem é o ponto de interseção entre a média de F1 e a média de F2 normalizadas, e as elipses pontilhadas são os desvios-padrão. Nas plotagens foram utilizadas as palavras-chave de Wells (1982): fleece [i:], kit [I], dress [ع], trap [æ], goose [u:], foot [ซ].
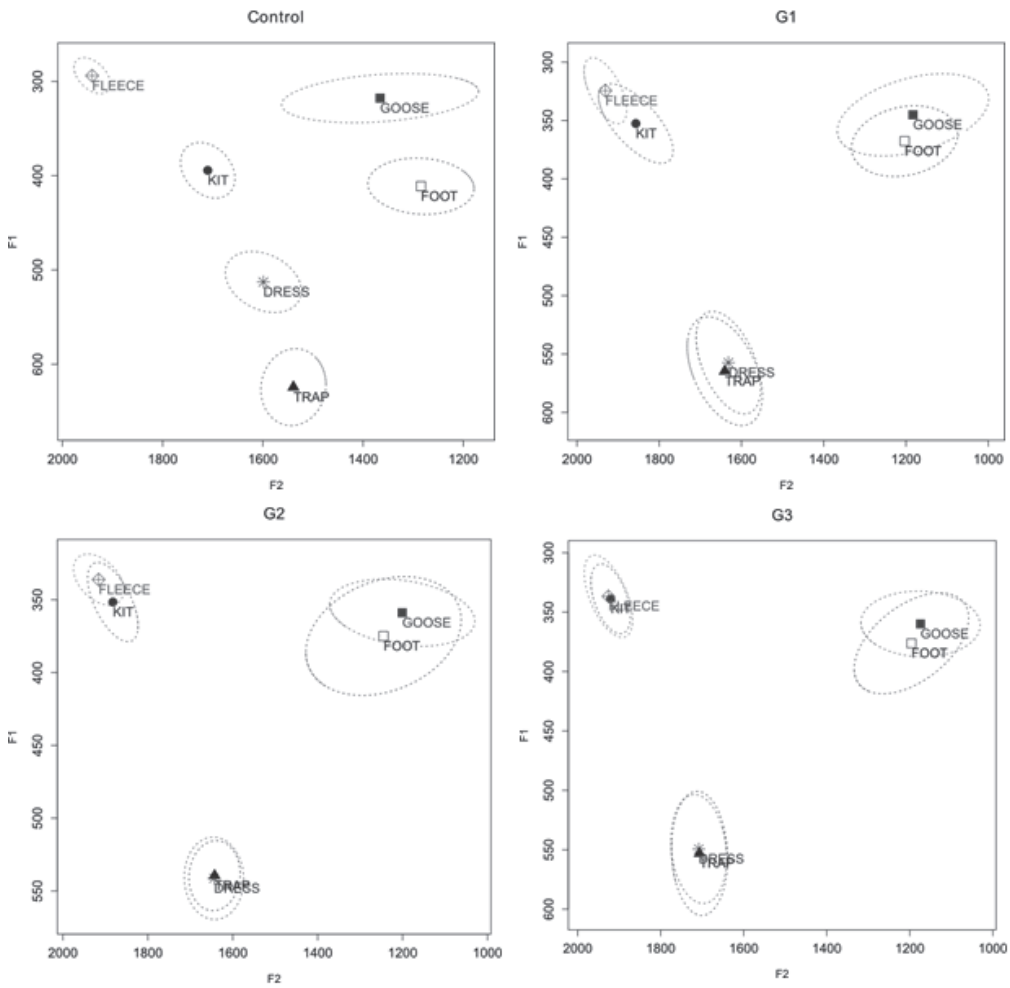

Fig. 4: Espaços vocálicos dos grupos de pesquisa

Com uma simples inspeção visual é possível constatar que os falantes nativos produziram as vogais-alvo em posições distintas e sem sobreposição dos desvios-padrão, o que era esperado por se tratar de vogais fonológicas produzidas por falantes nativos. Por outro lado, os alunos, independentemente do grupo, produziram os pares de vogais-alvo com sobreposição dos desvios-padrão e, em alguns casos, com sobreposição completa da vogal. A sobreposição indica áreas do espaço vocálico onde ambas as vogais são produzidas. É possível verificar nas plotagens que [i:] e [I] se aproximam cada Organon, Porto Alegre, v. 30, n. 58, p. 15-31, jan/jun. 2015. 
vez mais conforme a idade aumenta, com uma sobreposição total em G3. As vogais $[\varepsilon æ]$ dos alunos estão sobrepostas em todos os grupos, e [u: $\mho]$ mantêm uma distância relativamente constante nos três grupos de aprendizes.

Testes-t foram conduzidos para verificar se os pares de vogais são distinguidos pelos participantes em altura (F1) e em anterioridade/posterioridade (F2). Os resultados negritados indicam a ausência de contraste estatístico.

\begin{tabular}{|c|c|c|c|c|}
\hline \multirow{2}{*}{ Grupos } & \multirow{2}{*}{ Formante } & \multicolumn{3}{|c|}{ Resultado estatístico para os contrastes de F1 e F2 } \\
\hline & & [i:] vs $[\mathrm{I}]$ & {$[\varepsilon]$ vs $[æ]$} & [u:] vs [v] \\
\hline \multirow{2}{*}{ Controle } & F1 & $\mathrm{t}(238)=31, \mathrm{p}=0,000$ & $\mathrm{t}(236)=23, \mathrm{p}=0,000$ & $\mathrm{t}(237)=26, \mathrm{p}=0,000$ \\
\hline & $\mathrm{F} 2$ & $\mathrm{t}(238)=39, \mathrm{p}=0,000$ & $\mathrm{t}(236)=7, \mathrm{p}=0,000$ & $\mathrm{t}(237)=4, \mathrm{p}=0,000$ \\
\hline \multirow{2}{*}{ G1 } & F1 & $\mathrm{t}(238)=7, \mathrm{p}=0,000$ & $\mathrm{t}(233)=1,2, \mathrm{p}=0,220$ & $\mathrm{t}(238)=5, \mathrm{p}=0,000$ \\
\hline & $\mathrm{F} 2$ & $\mathrm{t}(238)=8, \mathrm{p}=0,000$ & $\mathrm{t}(233)=0,8, \mathrm{p}=0,425$ & $\mathrm{t}(238)=1, \mathrm{p}=0,324$ \\
\hline \multirow{2}{*}{ G2 } & F1 & $\mathrm{t}(237)=5, \mathrm{p}=0,000$ & $\mathrm{t}(238)=0,5, \mathrm{p}=0,595$ & $\mathrm{t}(237)=4, \mathrm{p}=0,000$ \\
\hline & $\mathrm{F} 2$ & $\mathrm{t}(237)=4, \mathrm{p}=0,000$ & $\mathrm{t}(238)=0,2, \mathrm{p}=0,869$ & $\mathrm{t}(237)=1,9, \mathrm{p}=0,06$ \\
\hline \multirow[t]{2}{*}{ G3 } & F1 & $t(232)=0,5, p=0,606$ & $t(236)=0,6, p=0,566$ & $\mathrm{t}(236)=4, \mathrm{p}=0,000$ \\
\hline & F2 & $\mathrm{t}(232)=0,8, \mathrm{p}=0,431$ & $\mathrm{t}(236)=0,2, \mathrm{p}=0,849$ & $t(236)=1, p=0,231$ \\
\hline
\end{tabular}

Fig. 5: Testes-t entre F1 e F2 das vogais contrastadas

De acordo com os testes-t, os falantes nativos distinguiram todos os pares de vogais tanto em F1 como em F2. Entre os alunos, nenhum grupo foi capaz de distinguir o par $[\varepsilon æ]$. Além disso, nenhum grupo foi capaz de distinguir [u: $\mho$ ] em F2, i.e. em anterioridade/posterioridade da língua. Com relação ao par [i: I], apenas o grupo G3 não foi capaz de distingui-lo.

A tabela a seguir apresenta as médias (em milissegundos) e desvios-padrão da duração das vogais por grupo.

\begin{tabular}{|c|c|c|c|c|c|c|}
\hline \multirow{2}{*}{ Grupo } & \multicolumn{7}{|c|}{ Duração das Vogais (em milissegundos) } \\
\cline { 2 - 7 } & {$[\mathrm{i}:]$} & {$[\mathrm{I}]$} & {$[\varepsilon]$} & {$[æ]$} & {$[\mathrm{u}:]$} & {$[\mathrm{J}]$} \\
\hline Controle & $90(\mathrm{DP}=23)$ & $73(\mathrm{DP}=24)$ & $87(\mathrm{DP}=26)$ & $124(\mathrm{DP}=37)$ & $105(\mathrm{DP}=34)$ & $83(\mathrm{DP}=28)$ \\
\hline G1 & $115(\mathrm{DP}=36)$ & $102(\mathrm{DP}=28)$ & $131(\mathrm{DP}=30)$ & $136(\mathrm{DP}=30)$ & $128(\mathrm{DP}=33)$ & $115(\mathrm{DP}=26)$ \\
\hline G2 & $106(\mathrm{DP}=38)$ & $99(\mathrm{DP}=30)$ & $128(\mathrm{DP}=39)$ & $134(\mathrm{DP}=33)$ & $117(\mathrm{DP}=37)$ & $112(\mathrm{DP}=38)$ \\
\hline G3 & $103(\mathrm{DP}=45)$ & $97(\mathrm{DP}=42)$ & $135(\mathrm{DP}=41)$ & $139(\mathrm{DP}=40)$ & $118(\mathrm{DP}=49)$ & $114(\mathrm{DP}=49)$ \\
\hline
\end{tabular}

Fig. 6: Médias e desvio-padrão (DP) da duração das vogais-alvo por grupo (em milissegundos)

Todos os grupos produziram vogais longas de fato mais longas que suas contrapartes breves, mas com uma diferença muito mais evidente no grupo controle do que nos grupos de aprendizes, e com um visível declínio dessa diferença conforme a idade de início de aquisição de cada grupo aumenta. A diferença entre [i: I] foi de $17 \mathrm{~ms}$ para o grupo controle contra $13 \mathrm{~ms}$ do grupo 
G1, 7ms de G2 e 6ms de G3. Entre [u: $v$ ], a diferença foi de 22ms para o grupo controle contra $13 \mathrm{~ms}$ de G1, $5 \mathrm{~ms}$ de G2 e $4 \mathrm{~ms}$ de G3. Apesar de [ $\varepsilon$ æ] não formar um par tenso-frouxo, pois ambas são frouxas, essas vogais também são distinguidas por falantes nativos pela duração. Há dialetos de inglês nos quais a distinção $[\varepsilon$ æ] é produzida apenas pela diferença de duração, e não por diferenças de qualidade espectral, como o Northern Cities Dialect (LADEFOGED, 2001), em áreas metropolitanas do nordeste dos Estados Unidos. Como pode ser visto, os falantes nativos produziram essas vogais com uma diferença média de $37 \mathrm{~ms}$, contra $5 \mathrm{~ms}$ de G1, $6 \mathrm{~ms}$ de G2 e $4 \mathrm{~ms}$ de G3.

Testes- $t$ também foram conduzidos para verificar se os pares de vogais são distinguidos pelos participantes em duração. Os resultados negritados indicam a ausência de contraste estatístico.

\begin{tabular}{|c|c|c|c|}
\hline \multirow{2}{*}{ Grupos } & \multicolumn{3}{|c|}{ Resultado estatístico para os contrastes de duração } \\
\hline & [i:] vs [I] & {$[\varepsilon]$ vs $[æ]$} & [u:] vs [v] \\
\hline Controle & $\mathrm{t}(238)=5,2, \mathrm{p}=0,000$ & $\mathrm{t}(236)=8,3, \mathrm{p}=0,000$ & $\mathrm{t}(237)=5,1, \mathrm{p}=0,000$ \\
\hline G1 & $\mathrm{t}(238)=3, \mathrm{p}=0,000$ & $\mathrm{t}(233)=1,3, \mathrm{p}=0,2$ & $\mathrm{t}(238)=3,5, \mathrm{p}=0,000$ \\
\hline G2 & $\mathrm{t}(237)=1,5, \mathrm{p}=0,125$ & $\mathrm{t}(238)=0,0, \mathrm{p}=0,987$ & $\mathrm{t}(237)=1,2, \mathrm{p}=0,233$ \\
\hline G3 & $\mathrm{t}(232)=1,2, \mathrm{p}=0,246$ & $\mathrm{t}(236)=0,7, \mathrm{p}=0,494$ & $\mathrm{t}(236)=0,7, \mathrm{p}=0,515$ \\
\hline
\end{tabular}

Fig. 7: Testes-t entre as médias de duração das vogais contrastadas

Os falantes nativos distinguiram todos pares de vogais pela duração; os participantes do grupo G1 distinguiram apenas os pares [i: r] e [u: $]$ ]; os outros dois grupos de aprendizes não produziram distinção em nenhum par com relação à duração das vogais.

Os valores de F1 e F2 foram também utilizados para a medição da distância euclidiana ${ }^{2}$ entre os pares de vogais. Para não serem influenciadas pelos valores de F2, que são naturalmente mais altos do que os de F1, as distâncias euclidianas foram calculadas com os valores da equação de normalização antes de serem transformados para $\mathrm{Hertz}^{3}$. A plotagem a seguir ilustra o conceito da distância euclidiana, mostrando que, entre os falantes nativos, o par cujas vogais estão mais distantes uma da outra é o [i: I], seguido por $[\varepsilon æ] \mathrm{e}$, finalmente, por [u: $\mho]$.

2 A distância euclidiana é uma medida de dissimilaridade que pode ser utilizada para medir a distância entre dois pontos em um sistema cartesiano. Sua fórmula é derivada do teorema de Pitágoras e, no caso desta pesquisa, foi calculada da seguinte forma:

Dist.Eucl. $=\sqrt{\left((\mathrm{F} 1 \mathrm{de} \mathrm{X}-\mathrm{F} 1 \mathrm{de} \mathrm{Y})^{2}+(\mathrm{F} 2 \text { de X-F2 de Y })^{2}\right)}$.

3 O método de normalização Watt e Fabricius (2002) resulta em valores que não estão em Hertz nem em Bark, mas em valores positivos e negativos relativos ao zero que representa a centroide do espaço vocálico, que podem ser posteriormente transformados para Hertz. Para o cálculo das Distâncias Euclidianas, foram utilizados os valores antes de serem transformados, assim, tanto F1 como F2 ficam em uma escala normalizada, não enviesada pelos valores mais altos de F2. Para facilitar a leitura, contudo, os resultados das distâncias euclidianas foram multiplicados por 1.000. 


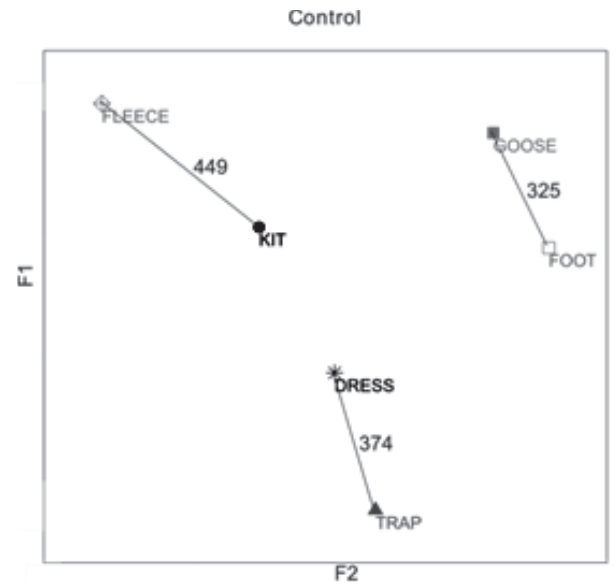

Fig. 8: Distâncias Euclidianas das vogais do grupo controle

A tabela a seguir apresenta as Distâncias Euclidianas entre as vogaisalvo para cada grupo.

\begin{tabular}{|c|c|c|c|}
\hline \multirow{2}{*}{ Grupos } & \multicolumn{3}{|c|}{ Distâncias Euclidianas entre vogais } \\
\cline { 2 - 4 } & {$[\mathrm{i}:]$ vs [I] } & {$[\varepsilon]$ vs [æ] } & {$[\mathrm{u}:]$ vs [v] } \\
\hline Controle & 449 & 374 & 325 \\
\hline G1 & 135 & 26 & 79 \\
\hline G2 & 67 & 6 & 78 \\
\hline G3 & 10 & 12 & 61 \\
\hline
\end{tabular}

Fig. 9: Distâncias Euclidianas entre vogais-alvo por grupo

Os valores das distâncias euclidianas diminuem com o aumento da idade, com exceção de [ $\varepsilon$ æ], que teve uma distância um pouco menor no G2 do que no G3. O par com maior distância entre os alunos foi o [i: I] para o G1 e o [u: $v]$ para os demais. O par com menor distância foi o $[\varepsilon æ]$ para os grupos G1 e G2, e o [i: I] para o G3. Contudo, nenhuma distância euclidiana dos aprendizes se aproximou das distâncias encontradas entre os falantes nativos. A maior distância entre vogais-alvo dos aprendizes foi de 135 para [i: I] do G1, que representa apenas 30\% da distância entre essas mesmas vogais do grupo controle. A tabela a seguir repete os valores da anterior, porém com a porcentagem de cada distância relativa à distância dos falantes nativos em parêntese. Com isso, é possível verificar que o par com a maior queda de Distância Euclidiana é o [ $\varepsilon$ æ]. 


\begin{tabular}{|c|c|c|c|}
\hline \multirow{2}{*}{ Grupos } & \multicolumn{3}{|c|}{ Distâncias Euclidianas entre vogais } \\
\hline & [i:] vs [I] & {$[\varepsilon]$ vs $[æ]$} & {$[\mathrm{u}:]$ vs $[\mathrm{U}]$} \\
\hline Controle & 449 & 374 & 325 \\
\hline G1 & $135(30 \%)$ & $26(7 \%)$ & $79(24 \%)$ \\
\hline G2 & $67(15 \%)$ & $6(2 \%)$ & $78(24 \%)$ \\
\hline G3 & $10(2 \%)$ & $12(3 \%)$ & $61(19 \%)$ \\
\hline
\end{tabular}

Fig. 10: Distâncias Euclidianas entre vogais-alvo por grupo, com a porcentagem de cada distância relativa à distância do grupo controle

Além dos contrastes entre os pares de vogais para cada grupo, é possível comparar as produções dos alunos com as produções dos falantes nativos. A figura 8 apresenta os espaços vocálicos dos falantes nativos e dos aprendizes em uma mesma plotagem, na qual é possível observar que a produção de cada par de vogais dos aprendizes está entre as vogais-alvo produzidas pelos falantes nativos.
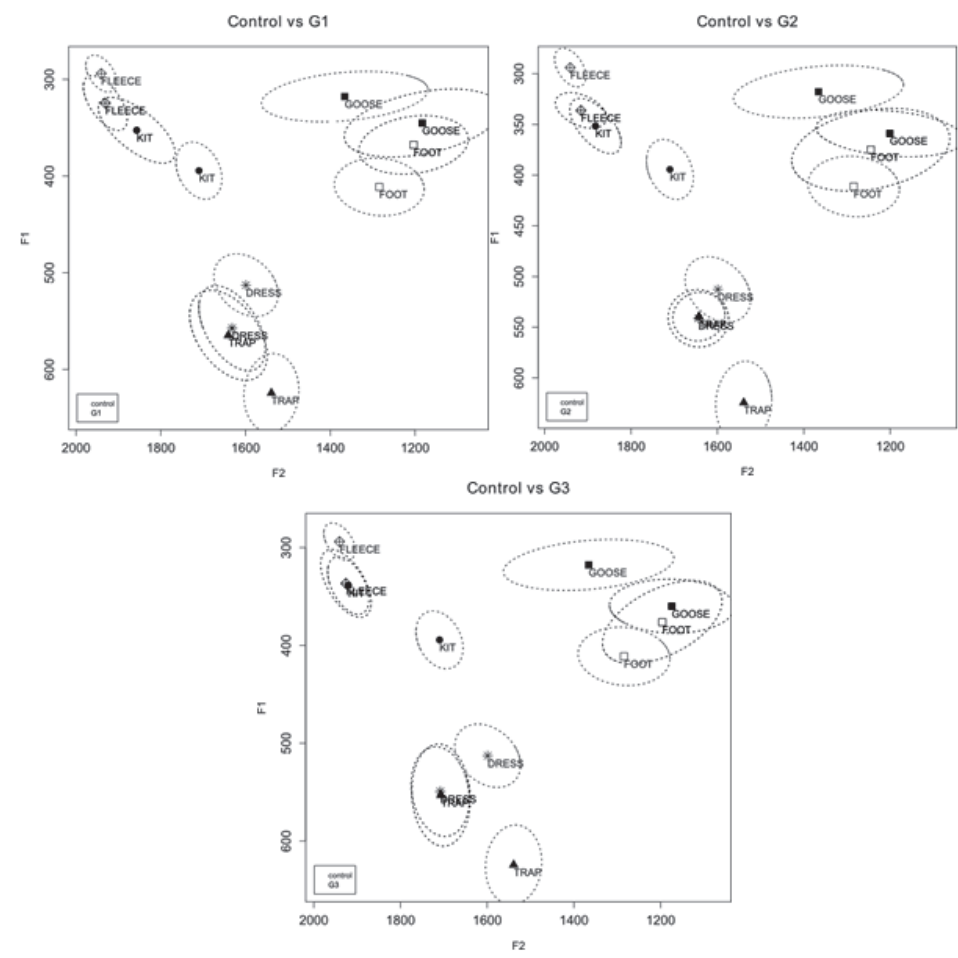

Fig. 11: Espaço vocálico do grupo controle comparado com os espaços vocálicos dos alunos Organon, Porto Alegre, v. 30, n. 58, p. 15-31, jan/jun. 2015. 
As vogais dos aprendizes tendem a ocupar uma posição intermediária entre as vogais-alvo dos falantes nativos. As vogais [u: $v$ ] são as mais constantes, pois, nos três grupos, elas foram produzidas entre [u: $v]$ dos falantes nativos, porém com valores menores de F2, i.e. mais posteriores. Além disso, o [ $\mho]$ dos alunos foi um pouco mais anterior do que [u:], o que não aconteceu entre os falantes nativos. As vogais [i: I] também foram produzidas entre as produções dos falantes nativos, porém com [I] diminuindo o seu valor de F1 e, consequentemente, aproximando-se de [i:] com o aumento da idade. Esse mesmo movimento pode ser observado em $[\varepsilon æ]$, que, apesar de sobrepostas, foram produzidas em posição intermediária por G1, mas aproximando-se de $[\varepsilon]$ com o aumento da idade. Esse último par também se mostrou um pouco mais anterior (valores maiores de F2) na produção dos alunos se comparada à dos falantes nativos.

Por último, foram calculadas as distâncias euclidianas entre cada vogal do grupo controle e essa mesma vogal dos grupos de alunos, apresentadas na tabela a seguir.

\begin{tabular}{|c|c|c|c|c|c|c|}
\hline \multirow{2}{*}{ Grupo } & \multicolumn{6}{|c|}{ Distância Euclidiana entre as vogais do grupo controle e as vogais dos grupos de alunos } \\
\cline { 2 - 7 } & {$[\mathrm{i}:]$} & {$[\mathrm{I}]$} & {$[\varepsilon]$} & {$[æ]$} & {$[\mathrm{u}:]$} & {$[\mathrm{\mho}]$} \\
\hline G1 & 111 & 238 & 153 & 237 & 258 & 178 \\
\hline G2 & 219 & 267 & 111 & 310 & 256 & 130 \\
\hline G3 & 167 & 332 & 188 & 322 & 288 & 163 \\
\hline
\end{tabular}

Fig. 12: Distâncias Euclidianas entre vogais do grupo controle e dos grupos de alunos

De maneira geral, as vogais dos alunos foram produzidas distantes das vogais dos falantes nativos. As vogais mais próximas às produções do grupo controle são [i:] de G1 e [ع] de G2, e as mais distantes são [I] e [æ] de G3. O grupo G1 foi o que mais se aproximou das vogais [i: I æ], mas foi o que mais se distanciou na produção de [ $\mho$. G2 foi o que mais se aproximou de [ $\varepsilon$ u: $v$ ], mas foi o que mais se distanciou de [i:]. O grupo G3 não foi o que mais se aproximou em nenhuma vogal, mas foi o que mais se distanciou em [I $\varepsilon$ æ u:].

\section{Discussão}

As análises acústicas mostraram uma grande queda de desempenho entre o grupo controle e o grupo G1, e quedas graduais entre os grupos G1, G2 e G3. 
A primeira análise foi a da distinção entre os pares de vogais para cada grupo. Enquanto as vogais dos falantes nativos ocuparam áreas claramente distintas no espaço vocálico F1-F2, os pares de vogais dos aprendizes tenderam a se sobrepor cada vez mais conforme as idades de início da aquisição aumentaram, mas com grande sobreposição já no grupo G1. As sobreposições das elipses de desvio-padrão no espaço vocálico refletem uma área na qual ambas as vogais são produzidas, o que pode proporcionar quebras de comunicação. Isso mostra que mesmo os aprendizes que começaram mais cedo ainda não tinham desenvolvido categorias fonológicas distintas para acomodar as vogais em suas interlínguas. Suas interlínguas sofrem os efeitos de atratores da L1, que conduzem a produção dos pares de vogais para apenas uma posição fonológica prototípica.

Apenas os grupos G1 e G2 produziram [i: I] com formantes estatisticamente distintos; por sua vez, nenhum grupo produziu $[\varepsilon æ]$ com distinção em F1 nem em F2. Contudo, os três grupos de alunos produziram [u: $v$ ] com diferença estatística em F1, mas não em F2. Isso quer dizer que [u:] foi estatisticamente mais alto do que [ $\mho]$, mas não suficientemente mais anterior; pelo contrário, o F2 de [u:] dos alunos teve valores menores, mostrando que o $[\mho]$ dos alunos tende a ser anteriorizado.

Essa distinção incompleta do par [u: $v$ ] dos alunos se dá pela natureza dinâmica e progressiva do processo de aquisição fonológica, proporcionando estágios intermediários de contraste, os contrastes encobertos ${ }^{4}$ (E.G. ECKMAN; IVERSON; SONG, 2014; SCOBBIE; GIBBON; WILLIAM, 2000), que podem ser estatisticamente relevantes, porém ainda não fortes o suficiente para serem percebidos. Isso não demonstra uma falha do processo de aquisição, muito pelo contrário, mostra o sistema buscando um estado crítico para se mover de um ponto de atração (baseado na fonologia da L1) para outro (baseado na fonologia da L2).

$\mathrm{O}$ par $[\varepsilon æ]$ foi o que apresentou maior dificuldade aos alunos, com sobreposição já no grupo G1. A dificuldade maior entre $[\varepsilon æ]$ também foi encontrada por Baptista (2000), Bion et al. (2006), Rauber (2006) e Barboza (2008), mesmo com seus participantes sendo alunos universitários de Letras e/ou professores de inglês com vasta experiência.

É reconhecido que as vogais $[\varepsilon æ]$ estão se aproximando em qualidade formântica mesmo entre falantes nativos, como no já mencionado Northern Cities Dialect (LADEFOGED, 2001), entre outros. Contudo, nesses dialetos as vogais continuam sendo claramente distintas por causa da du-

4 Covert Contrast.

Organon, Porto Alegre, v. 30, n. 58, p. 15-31, jan/jun. 2015. 
ração, que é mais longa em [æ] do que em [ع]. Dois participantes do grupo controle cresceram em cidades do Northern Cities Dialect e, de fato, produziram $[\varepsilon æ]$ com Distâncias Euclidianas mínimas; contudo, estão entre os participantes que tiveram maior diferença na duração de $[\varepsilon æ], 57 \mathrm{~ms}$ e $43 \mathrm{~ms}$. Já os aprendizes não produziram contraste na duração nem na qualidade formântica dessas vogais. A maior diferença de duração entre os alunos foi de $18 \mathrm{~ms}$ (G1H05), e a média da diferença de duração dessas vogais entre os aprendizes foi de apenas $5 \mathrm{~ms}$.

Ainda sobre os contrastes das vogais em cada grupo, o cálculo das distâncias euclidianas entre as vogais de cada par permitiu verificar que os falantes nativos produziram pares de vogais significativamente mais separadas do que os aprendizes de qualquer grupo. O par mais separado dos aprendizes ([i: de G1) não obteve uma distância superior a 30\% da distância do grupo controle. As distâncias encontradas para G2 e G3 fortalecem a hipótese de tendência de aumento da dificuldade de aquisição com o aumento da idade, pois as distâncias do grupo G2 foram menores do que as de G1, e as de G3 menores do que as de G2.

A conclusão de queda abrupta entre falantes nativos e aprendizes mais novos e quedas graduais entre os aprendizes é reforçada pela análise de duração das vogais. A diferença na duração das vogais de cada par diminuiu já entre o grupo controle e G1, e continuou diminuindo entre G1 e G2, e entre G2 e G3. Isso mostra, mais uma vez, que os aprendizes têm dificuldade em criar categorias fonológicas distintas para os novos gestos vocálicos em questão. Por causa da força dos atratores de suas interlínguas, que são influenciados pela L1, os aprendizes tendem a produzir as duas vogais de cada par em uma única posição fonológica prototípica.

A última análise, da distância entre uma vogal do grupo controle e essa mesma vogal de cada grupo de aprendizes, foi a única que não demonstrou vantagem para o grupo de aprendizes mais novos. O grupo G1 foi o que mais se aproximou em apenas três vogais [i: I æ], e o que mais se aproximou das ouras três, $[\varepsilon$ u: $v$ ], foi o grupo G2. Ademais, tanto G1 como G2 tiveram uma vogal cada que foi a mais distante da produção do grupo controle, [v] e [i:], respectivamente. Analisando-se os dados de G1 e G2 conjuntamente em comparação aos dados de G3, é possível manter a hipótese de aumento da dificuldade de aquisição com o aumento da idade, mas mais do que isso, essa análise mostra que ter pares de vogais mais bem contrastadas em F1 e F2 e em duração não garante que essas vogais estão mais próximas das produções dos falantes nativos. Essa conclusão corrobora a natureza dinâmica e progressiva da aquisição, bem como os estágios 
intermediários de distinção fonológica, como os contrastes encobertos já mencionados. Os sistemas fonológicos dos aprendizes precisam se mover não apenas para posições de contraste, mas para posições de contraste próximas às do grupo controle.

\section{Conclusões}

O objetivo deste estudo foi o de investigar como o fator "idade do início da aquisição" pode influenciar a aquisição das vogais [i: i $\varepsilon$ æ u: ] por aprendizes brasileiros que começaram seus cursos de inglês em diferentes idades exclusivamente em salas de aula do Brasil. Esse objetivo foi alcançado e a hipótese, da tendência de aumento da dificuldade de aquisição das vogais-alvo com o aumento da idade no início da aquisição, foi confirmada pelos dados. Ou seja, com o aumento da idade de início dos estudos de inglês, as vogais de cada par tenderam a ser menos separadas entre si, menos contrastadas em duração, e mais distantes da produção dos falantes nativos. Contudo, o fator idade não se mostrou fator determinante na aquisição das vogais, pois mesmo a produção do grupo de aprendizes mais novos foi muito díspar da do grupo controle.

\section{BIBLIOGRAFIA}

ARANTES, P. Duration_multiple.praat 2008.

ARANTES, P. Formants.praat 2010.

ARANTES, P. Collectformants.praat 2011.

BAPTISTA, B. O. The acquisition of English vowels by Brazilian-Portuguese speakers. Pós-Graduação em Inglês, Universidade Federal de Santa Catarina, 2000. ISBN 858659301X.

BARBOZA, C. Descrição acústica dos sons vocálicos anteriores do inglês e do português realizados por professores de inglês língua estrangeira no oeste potiguar. 183 f. 2008. Dissertação (Mestrado Acadêmico em Linguística Aplicada)-Universidade Estadual do Ceará, Fortaleza BIALYSTOK, E.; HAKUTA, K. Confounded age: Linguistic and cognitive factors in age differences for second language acquisition. Second language acquisition and the critical period hypothesis, p. 161-181, 1999. BION, R. A. H. et al. Category formation and the role of spectral quality in the perception and production of English front vowels. Interspeech, 2006. Citeseer. 
BOERSMA, P.; WEENINK, D. PRAAT 2011.

BONGAERTS, T. Introduction: Ultimate attainment and the critical period hypothesis for second language acquisition. International Review of Applied Linguistics in Language Teaching, v. 43, n. 4, p. 259-267, 2005. BYBEE, J. Phonology and language use. Cambridge University Press, 2003. ISBN 0521533783.

CELCE-MURCIA, M.; BRINTON, D. M.; GOODWIN, J. M. Teaching pronunciation: a reference for teachers of English to speakers of other languages. Cambridge University Press, 1996.

CRISTÓFARO-SILVA, T. Descartando fonemas: a representação mental da fonologia de uso. In: HORA, D. e COLLISCHONN, G. (Ed.). Teoria linguística: fonologia e outros temas. João Pessoa: Editora Universitária, 2003. ECKMAN, F. R.; IVERSON, G. K.; SONG, J. Y. Covert contrast in the acquisition of second language phonology. Perspectives on Phonological Theory and Development: In honor of Daniel A. Dinnsen, v. 56, p. 25, 2014. ELLIS, R. The study of second language acquisition. Oxford: Oxford University Press, 2008.

FLEGE, J. E. Second language speech learning: Theory, findings, and problems. In: STRANGE, W. (Ed.). Speech perception and linguistic experience: Issues in cross-language research. Baltimore: York Press, 1995. p.233-277. FLEGE, J. E. Age of learning and second language speech. In: BIRDSONG, D. (Ed.). Second language acquisition and the critical period hypothesis. Mahwah: Lawrence Erlbaum Associates, 1999. p.101-131. FLEGE, J. E. Language contact in bilingualism: Phonetic system interactions. Laboratory phonology, v. 9, p. 353-382, 2007.

HAKUTA, K. A critical period for second language acquisition? In: BAILEY JR, D. B.;BRUER, J. T., et al (Ed.). Critical thinking about critical periods. Baltimore, Maryland: Paul H. Brookes Publishing Co., 2001. cap. 10, p.193-205.

KELLY, G. How to teach pronunciation. Pearson Education India, 2006. KENDALL, T.; THOMAS, E. R. Vowels: Vowel manipulation, normalization, and plotting in R. R package, version. 12009.

KUHL, P. K. Human adults and human infants show a "perceptual magnet effect" for the prototypes of speech categories, monkeys do not. Perception \& psychophysics, v. 50, n. 2, p. 93-107, 1991.

KUHL, P. K. Innate predispositions and the effects of experience in speech perception: The native language magnet theory. In: BOYSSIB-

BARDIES, B. (Ed.). Developmental neurocognition: Speech and face processing in the first year of life. The Hague: Kluwer Academic Publishers, 1993. p.259-274. ISBN 9048142512. 
KUHL, P. K. et al. Phonetic learning as a pathway to language: new data and native language magnet theory expanded (NLM-e). Philosophical Transactions of the Royal Society B: Biological Sciences, v. 363, n. 1493, p. 979-1000, 2008.

LADEFOGED, P. Vowels and consonants: an introduction to the sounds of languages. Massachusetts: Blackwell, 2001.

LEATHER, J. Phonological acquisition in multilingualism. In: MAYO, M. D. P. G. e LECUMBERRI, M. L. G. (Ed.). Age and the Acquisition of English as a Foreign Language. Clevedon: Multilingual Matters, 2003. cap. 2, p.23-58.

MOYER, A. Age, accent, and experience in second language acquisition: an integrated approach to critical period inquiry. Multilingual Matters, 2004. PARRINO, A. The politics of pronunciation and the adult learner. Adult ESL: Politics, Pedagogy and Participation in Classroom and Community Programs, p. 171-184, 1998.

R CORE TEAM. R: A language and environment for statistical computing 2012.

RAUBER, A. S. Perception and production of English vowels by Brazilian EFL speakers. 2006. (Tese de Doutorado). Universidade Federal de Santa Catarina, Florianópolis.

SCOBBIE, J. M.; GIBBON, F.; WILLIAM, J. Covert contrast as a stage in the acquisition of phonetics and phonology. Papers in laboratory phonology V: Acquisition and the lexicon, v. 5, p. 194, 2000.

SINGLETON, D. M.; RYAN, L. Language acquisition: the age factor. Multilingual Matters, 2004.

VALLABHA, G. K.; TULLER, B. Systematic errors in the formant analysis of steady-state vowels. Speech communication, v. 38, n. 1, p. 141-160, 2002. WATT, D.; FABRICIUS, A. Evaluation of a technique for improving the mapping of multiple speakers' vowel spaces in the F1 F2 plane. Leeds Working Papers in Linguistics and Phonetics, v. 9, n. 9, p. 159-173, 2002. WELLS, J. C. Accents of English. Cambridge University Press, 1982. ISBN 0521297192.

WEMPE, T.; BOERSMA, P. The interactive design of an F0-related spectral analyser. Proc. 15th ICPhS, 2003. p.343-346.

Recebido em: 28/11/2014. Aceito em: 11/03/2015. 